\title{
Cost Effectiveness of Sequencing 34 Cancer-Associated Genes as an Aid for Treatment Selection in Patients with Metastatic Melanoma
}

\author{
Yonghong $\mathrm{Li}^{1} \cdot$ Lance A. Bare $^{1} \cdot$ Richard A. Bender $^{1} \cdot$ John J. Sninsky ${ }^{1}$. \\ Leslie S. Wilson ${ }^{2,3} \cdot$ James J. Devlin ${ }^{1} \cdot$ Frederic M. Waldman $^{1}$
}

Published online: 30 April 2015

(c) The Author(s) 2015. This article is published with open access at Springerlink.com

\begin{abstract}
Objective To determine whether a next-generation sequencing (NGS) panel of 34 cancer-associated genes would cost-effectively aid in the treatment selection for patients with metastatic melanoma, compared with a single-site $B R A F$ V600 mutation test.

Methods A decision model was developed to estimate the costs and health outcomes of the two test strategies. The cost effectiveness of these two strategies was analyzed from a payer perspective over a 2-year time horizon with model parameters taken from the literature.

Results In the base case, the gene sequencing panel strategy resulted in a cost of US $\$ 120,022$ and 0.721 quality-adjusted life years (QALYs) per patient, whereas the single-site mutation test strategy resulted in a cost of US\$128,965 and 0.704 QALYs. Thus, the gene sequencing panel strategy cost US\$8943 less per patient and increased QALYs by 0.0174 per patient. Sensitivity analyses showed that, compared with the single-site mutation test strategy, the gene sequencing panel strategy had a $90.9 \%$ chance of having reduced costs and increased QALYs, with the cost of the gene sequencing panel test having minimal effect on the incremental cost.
\end{abstract}

Electronic supplementary material The online version of this article (doi:10.1007/s40291-015-0140-9) contains supplementary material, which is available to authorized users.

Yonghong Li

yonghong.li@celera.com

1 Quest Diagnostics, Alameda and San Juan Capistrano, Alameda, CA 94502, USA

2 Department of Medicine, University of California, San Francisco, CA 94143, USA

3 Department of Pharmacy, University of California, San Francisco, CA 94143, USA
Conclusion Compared with the single-site mutation test, the use of an NGS panel of 34 cancer-associated genes as an aid in selecting therapy for patients with metastatic melanoma reduced costs and increased QALYs. If the base-case results were applied to the 8900 patients diagnosed with metastatic melanoma in the USA each year, the gene sequencing panel strategy could result in an annual savings of US\$79.6 million and a gain of 155 QALYs.

\section{Key Points}

Genetic tests of tumors are used to inform treatment selection for patients with metastatic melanoma. A gene sequencing panel test can interrogate mutations in multiple cancer-associated genes, while a singlesite mutation test determines the genotype of a single variant.

From a US health-care payer perspective, testing and selecting first-line targeted treatment for metastatic melanoma using a next-generation sequencing panel of 34 cancer-associated genes can lower the medical costs and increase the patient's quality and length of life, compared with a single-site mutation test. Thus, the gene sequencing panel test merits consideration in the clinical management of patients with metastatic melanoma.

\section{Introduction}

Melanoma is one of the most common cancers in the USA, with an estimated 76,690 newly diagnosed cases and 9480 deaths annually [1]. About $2-5 \%$ of newly diagnosed 
melanomas present with metastatic disease [2]. Prior to recently approved therapies, patients with metastatic melanoma generally had a poor prognosis, with a median survival time of 6-9 months and a 5-year survival of less than $15 \%[3,4]$. Newer therapies report improved survival times [5-7].

For patients with metastatic melanoma, the National Comprehensive Cancer Network (NCCN) recommends systemic therapy, enrollment in a clinical trial, or best supportive care [2]. Systemic therapy can improve patient survival and includes immunotherapies and targeted therapies. The approved immunotherapies are ipilimumab for first-line treatment and pembrolizumab and nivolumab for second-line treatment. Ipilimumab binds CTLA-4 (cytotoxic $\mathrm{T}$ lymphocyte-associated antigen 4), thereby blocking the inhibition of cytotoxic $\mathrm{T}$ lymphocytes by CTLA-4 and consequently enabling cytotoxic $\mathrm{T}$ lymphocytes to recognize and destroy cancer cells. Although ipilimumab can elicit long-lasting antitumor effects, it has a relatively low response rate $(28 \%)$ and can cause serious adverse events [5]. Several targeted therapies have also been approved for patients with metastatic melanoma [8]. For example, the BRAF kinase inhibitor vemurafenib is a first-line treatment option for patients carrying a $B R A F$ V600E mutation. Clinical studies have also demonstrated that patients with activating mutations in KIT may respond to therapy with imatinib, an inhibitor of tyrosine kinase receptors [9-11]. In addition, patients with other mutations may be candidates for treatment with therapies approved for use in tumors of different origins or newer therapies that are being evaluated in clinical trials for metastatic melanoma $[12,13]$.

Approved systemic therapies are costly-a course of ipilimumab therapy, for example, can cost as much as US\$150,000 [14] —and no definitive predictive biomarkers are available to identify which patients are most likely to benefit from ipilimumab treatment. For targeted therapy with BRAF or KIT inhibitors, patients who carry specific tumor mutations are more likely to respond, and these mutations can be identified by genetic tests prior to initiation of therapy. Using a genetic test to choose the most appropriate drugs may not only help to direct patients to appropriate therapies sooner but also save money by avoiding therapies that are less likely to be effective.

The FDA has approved a companion single-site mutation test for vemurafenib, the cobas ${ }^{\circledR} 4800 B R A F$ V600 mutation test [15], which is a real-time polymerase chain reaction (PCR) assay designed to detect the $B R A F$ V600E (T1799A) mutation. However, this test may not identify some patients who are likely to respond to BRAF inhibitors-for example, patients who carry other $B R A F$ mutations such as V600K, V600E2, L597Q, L597S, and K601E. Tumors harboring these $B R A F$ mutations are likely to respond to inhibitors of BRAF and MEK [mitogen-activated protein (MAP)/extracellular signal-regulated kinase (ERK) kinase] based on data from pre-clinical and individual patient studies as well as from clinical trials $[6,16$, 17]. Furthermore, the single-site $B R A F$ mutation test does not interrogate the KIT gene for mutations such as L576P that respond to imatinib therapy [18].

Next-generation sequencing (NGS) provides a platform for the simultaneous identification of mutations in multiple genes that are known to harbor hotspot mutations in tumors. The comprehensive molecular overview of the patient's tumor should allow more informed therapy decisions for genetically heterogeneous diseases such as melanoma. Research-use-only and laboratory-developed NGS tests are available to sequence multiple cancer-associated genes. As an example, the laboratory-developed OncoVantage $^{\mathrm{TM}}$ gene sequencing panel test uses NGS to interrogate the most commonly mutated exons in 34 cancer-associated genes, including $B R A F, K I T$, and other genes that can contain clinically actionable mutations in melanoma tumors [13] (Supplementary Table 1). Therefore, this test could be used to identify patients with KIT mutations who may benefit from imatinib therapy, patients with $B R A F$ V600 mutations who may benefit from vemurafinib, or patients with $B R A F$ mutations not readily detectable by the single-site mutation test (e.g. V600K, V600E2, L597S and K601E) who may benefit from both BRAF and MEK inhibitors.

However, which test scenario is the most cost-effective method of helping to identify the optimal treatment for patients with metastatic melanoma is unknown. In this study, we used clinical study results to model the cost effectiveness of the 34-gene NGS panel test and the cobas ${ }^{\circledR}$ $B R A F$ V600 single-site mutation test for guiding therapy selection in patients with metastatic melanoma.

\section{Methods}

\subsection{Model}

The aim of this study was to compare the cost effectiveness of two gene test strategies that guide the selection of therapies for patients with metastatic melanoma. A schematic of the decision tree for the two test-and-treat strategies is shown in Fig. 1. One branch of the decision tree starts with a 34-gene sequencing panel test and the other the cobas ${ }^{\circledR}$ BRAF V600 single-site mutation test. Treatments in each strategy are based on the NCCN guidelines for melanoma [2]. In both strategies, patients who test positive for BRAF V600 mutations received vemurafenib. BRAF V600 mutations considered positive included V600E and V600K for the single-site mutation test 
Fig. 1 Schematic of the decision tree (a) and partitioned survival model (b). NGS nextgeneration sequencing
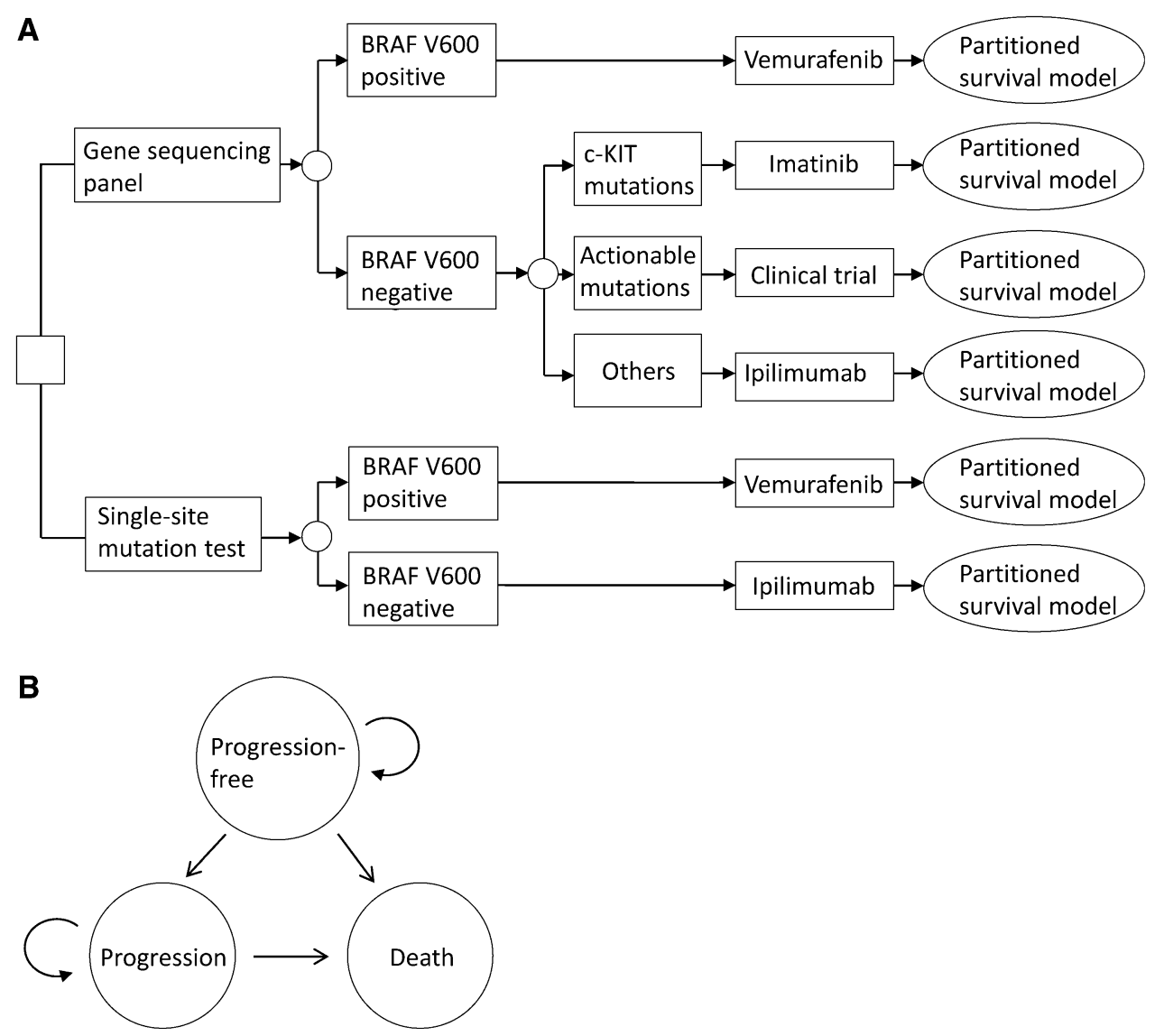

and V600E, V600E2, V600K, V600R, and V600D for the gene sequencing panel. These BRAF V600 mutations were sensitive to vemurafenib in cell culture studies [16], and patients who test positive for these mutations responded to vemurafenib in clinical trials [6, 19]. Patients who test negative for BRAF V600 mutations by the single-site mutation test received ipilimumab. Patients who test negative for $B R A F$ V600 mutations by the gene sequencing panel received one of three therapies: (1) imatinib if they carried KIT mutations, (2) ipilimumab if they carried no actionable mutations, or (3) were enrolled in a clinical trial if they carried other actionable mutations. Clinical trials that do not enroll patients based on a genetic marker were not included in the model because these trials would probably affect the two strategies equally.

The possible health outcomes from these treatments comprised three mutually exclusive states: progression-free survival, progression, and death. Transitions between states occurred at 3-month intervals over a 2-year time horizon. In the partitioned survival model, patients entered the study in the initial state of progression-free survival and at each cycle, either remained in that state or transitioned to one of the other two states. Patients could remain in the progression state or transition to the death state in subsequent cycles.

\subsection{Costs and Utilities}

Costs for the tests were taken from the Medicare clinical laboratory fee schedule [20] and Quest Diagnostics. Costs for drugs were calculated using the average weighted price (AWP) in the Redbook and dosages provided in the vemurafenib and ipilimumab prescribing information and the Carvajal et al. [11] study. Since this cost-effectiveness study was conducted from a payer's perspective, the payer would have no drug costs for the clinical trial, which would be covered by the sponsoring pharmaceutical company. Health utilities for progression-free survival, progression, and death were taken from Beusterien et al. [21].

\subsection{Distribution of Patient Populations}

The proportion of patients who were $B R A F$ V600 positive was based on BRAF mutation analyses from 295 consecutive de-identified formalin-fixed, paraffin-embedded (FFPE) tissue specimens submitted to Quest Diagnostics for testing [22] and from 496 clinical trial patients reported in the single-site mutation test package insert [15]. These analyses were conducted using both the single-site mutation test and Sanger sequencing, which detects more BRAF V600 E/K mutations as well as other BRAF mutations (e.g. 
V600 R/G), and any drop-outs in the testing were not included in the calculation. For this cost-effectiveness study, NGS was assumed to be equivalent to the Sanger sequencing in both assay sensitivity and specificity.

In the base-case analysis, we assumed that among patients who test negative for BRAF V600 mutations, $10 \%$ were enrolled in clinical trials, $6.6 \%$ received imatinib, and $83.4 \%$ received ipilimumab. Approximately $3-5 \%$ of adults with cancer in the USA join clinical trials [23]; since patients with metastatic melanoma have a poor prognosis and a limited choice of therapies, we assumed that $10 \%$ would be a reasonable base-case estimate for the proportion of patients enrolled in clinical trials when they did not carry a BRAF V600 mutation. The impact of this assumption was investigated in the sensitivity analysis.

Since co-occurrence of BRAF and KIT mutations is rare [11], we assumed that the rate of KIT mutations in the $B R A F$ negative patient population would be $6.6 \%$ (27/ 409)_an NGS analysis of tumors from 699 patients with advanced melanoma found that 27 patients carried a $K I T$ mutation and 409 patients did not carry a $B R A F$ mutation [24].

\subsection{Clinical Parameters for the Partitioned Survival Models}

Partitioned survival analysis uses empirical clinical data for overall survival and progression-free survival to determine the number of patients in the three states of the model [25, 26]. Estimates of progression-free survival $\left(P_{\mathrm{PFS}}\right)$ and overall survival $\left(P_{\mathrm{OS}}\right)$ were based on results from phase 3 clinical trials, with separate estimates for each 3 months segment of the study. Specifically, the values for $P_{\mathrm{PFS}}$ and $P_{\text {Os }}$ were taken from the midpoint of each 3-month interval from the Kaplan-Meier survival curves reported in the clinical trials of vemurafenib [6], ipilimumab [5], and imatinib [11] (Supplementary Table 2). The total number of patients in a particular cycle was then calculated as $\left(n \times P_{\mathrm{PFS}}\right)$ in the progression-free state, as $\left[n \times\left(P_{\mathrm{OS}}-\right.\right.$ $\left.\left.P_{\mathrm{PFS}}\right)\right]$ in the progression state, and as $\left[n \times\left(1-P_{\mathrm{OS}}\right)\right]$ in the death state (where $n$ was the initial number of patients). For the outcome of patients enrolled in the clinical trials, it was assumed that the average response would be the same as that for ipilimumab therapy, the likely treatment in the comparator arm of a clinical trial for patients who are not eligible for vemurafenib therapy.

\subsection{Base-Case Analysis}

Base-case outcomes were total costs incurred by the payer and QALYs, where both were calculated on a per-patient basis over a time horizon of 2 years. Incremental costs, incremental effectiveness, and incremental cost-effectiveness ratio were calculated to compare the two test strategies. Costs were discounted at $3 \%$ per year [27]. Calculations of the base-case outcomes were done using Microsoft Office Excel 2010.

The total costs comprised a one-time genetic test and the sum of the drug costs incurred in all 3-monthly cycles over 2 years. For each cycle, the cost of the drugs was calculated by multiplying the cost of drugs per patient and the number of patients eligible to receive therapy at the midpoint of the cycle as estimated in the partitioned survival models. For treatment with vemurafenib and imatinib, patients received medication as long as they remained in the progression-free survival state. For ipilimumab therapy, we used the FDA-approved regimen, which is the administration of the drug every 3 weeks for a total of 4 doses. The NCCN guidelines also allow the reinduction of ipilimumab for patients who relapse after initial response or progress after stable disease for more than 3 months if they did not experience significant toxicity during prior ipilimumab therapy [2]. And since Barzey et al. [28] reported that an average of $7 \%$ of the patients received 5.44 doses of the drug, total costs for ipilimumab included both costs for the eligible patients in the midpoint of the first cycle and additional costs for re-induction.

The overall effectiveness was the sum of effectiveness calculated for each of the 3-month cycles over 2 years. The effectiveness in a specific cycle was calculated as the sum of (1) the health utility for progression-free survival multiplied by the number of patients in the progression-free survival state at the midpoint of the cycle and (2) the health utility for progression multiplied by the number of patients at the progression state at the midpoint of the cycle.

\subsection{Sensitivity Analysis}

The sensitivity of the calculated costs and effectiveness to uncertainties in the parameter estimates was examined using @Risk software (version 6.1, Palisade Corporation) for Microsoft Office Excel 2010. The ranges for re-induction and health-state utilities were taken from Barzey et al. [28]. The range of relative health outcomes for clinical trials or ipilimumab therapy was set at $\pm 50 \%$ from the base value, and the range of all other variables was set at $\pm 25 \%$ from the base values. A triangular distribution was assumed for the defined ranges of all parameters. The probabilistic sensitivity analysis was based on 5000 Monte Carlo simulations to examine the effect of simultaneously varying all variables in the defined ranges on incremental cost and incremental effectiveness. 


\section{Results}

\subsection{Base Case}

The base-case analysis used the $B R A F$ mutation frequency observed in a study of melanoma samples submitted to a large national testing laboratory [22] ( 0.429 by the gene sequencing panel and 0.349 by the single-site mutation test; Table 1). The single-site mutation test strategy and the gene sequencing panel strategy resulted in a cost to payers of US $\$ 128,965$ and US $\$ 120,022$ per patient, respectively, over a 2-year time horizon (Table 2). Therefore, the gene sequencing panel strategy was associated with a cost savings of US $\$ 8943$ per patient over the single-site mutation test strategy. The QALYs resulting from the single-site mutation test strategy and the gene sequencing panel strategy were 0.704 and 0.721 per patient, respectively, over the same time horizon. Therefore, the gene sequencing panel strategy provided an incremental 0.0174 QALYs over the single-site mutation test strategy. These results suggest that the gene sequencing panel strategy is a dominant (less expensive and more effective) strategy over the single-site mutation test strategy for patients with metastatic melanoma.

\subsection{Sensitivity Analyses}

Since a higher mutation frequency was observed when screening melanoma patients for enrollment in a clinical study of vemurafenib, we also examined cost-effectiveness using the BRAF mutation frequencies reported in that clinical trial [15] (0.541 test positives for the single-site mutation test and 0.617 test positives for the gene sequencing panel). In this analysis, the gene sequencing panel strategy was associated with a cost savings of US\$5888 and increased QALYs of 0.0157 , compared with the single-site mutation test strategy (Supplementary Table 3). Thus, the gene sequencing panel strategy remained dominant.

When the effects of uncertainties in the parameter estimates on incremental costs were investigated in multivariable sensitivity analysis, the cost of ipilimumab had the largest effect, followed by the proportion of patients who test positive by the single-site mutation test, the cost of

Table 1 Model parameters and values tested in base-case and sensitivity analyses

\begin{tabular}{|c|c|c|c|}
\hline Parameter & Base-case & Range $^{\mathrm{a}}$ & References \\
\hline \multicolumn{4}{|l|}{ Proportions of patients } \\
\hline$B R A F$ mutation-positive by single-site mutation test & 0.349 & $0.262-0.436$ & [22] \\
\hline$B R A F$ mutation-positive by gene sequencing panel & 0.429 & $0.322-0.536$ & {$[22]^{\mathrm{b}}$} \\
\hline Gene sequencing panel $B R A F$ mutation-negatives and receiving imatinib & 0.066 & $0.050-0.083$ & Assumption \\
\hline Gene sequencing panel $B R A F$ mutation-negatives and enrolled in clinical trials & 0.1 & $0.075-0.125$ & Assumption \\
\hline \multicolumn{4}{|l|}{ Reinduction of ipilimumab } \\
\hline Reinduction rate & $7 \%$ of patients & $5 \%-7 \%$ & [28] \\
\hline Number of doses & 5.44 & $4.35-5.76$ & [28] \\
\hline \multicolumn{4}{|l|}{ Costs } \\
\hline Vemurafenib (3 months) & US\$39,063 & US $\$ 29,297-48,829$ & [14] \\
\hline Ipilimumab (4 doses) & US\$150,227 & US $\$ 112,670-187,784$ & [14] \\
\hline Imatinib (3 months) & US\$66,316 & US $\$ 49,737-82,895$ & [14] \\
\hline Single-site mutation test & US\$179 & US\$134-224 & [20] \\
\hline Gene sequencing panel test & US\$2400 & US\$1800-3000 & $\begin{array}{l}\text { Quest } \\
\text { diagnostics }^{\mathrm{c}}\end{array}$ \\
\hline \multicolumn{4}{|l|}{ Health state utilities } \\
\hline Progression free & 0.8 & $0.64-0.96$ & [21] \\
\hline Progression & 0.52 & $0.42-0.62$ & [21] \\
\hline Death & 0 & - & - \\
\hline Relative health outcomes of clinical trial versus ipilimumab therapy & 1 & $0.5-1.5$ & Assumption \\
\hline Cost discount & $3 \%$ per year & - & [27] \\
\hline
\end{tabular}

${ }^{a}$ The ranges for reinduction and health state utilities were from reference [28]; the upper and lower bounds were $\pm 50 \%$ of base values for relative health outcomes of clinical trials versus ipilimumab therapy and $\pm 25 \%$ of base values for other variables

b Also assuming equivalency of next-generation sequencing and Sanger sequencing in the detection of mutations

${ }^{c}$ Expected costs for test will vary based on individual health plan and government payer rates and coverage policies 
Table 2 Base-case outcome

\begin{tabular}{lllll}
\hline Strategy & $\begin{array}{l}\text { Cost } \\
\text { (US\$/patient) }\end{array}$ & $\begin{array}{l}\text { Incremental cost } \\
\text { (US\$patient) }\end{array}$ & $\begin{array}{l}\text { Effect } \\
\text { (years/patient) }\end{array}$ & $\begin{array}{l}\text { Incremental effect }_{(\text {years/patient) }} \\
\text { ICER }\end{array}$ \\
\hline Single-site mutation test & 128,965 & Reference & 0.704 & Reference \\
Gene sequencing panel & 120,022 & -8943 & 0.721 & 0.0174 \\
\hline
\end{tabular}

${ }^{a}$ Incremental cost effectiveness ratio (US\$ per quality-adjusted life year gained)

vemurafenib, and the proportion of patients in clinical trials (Fig. 2a). Compared with these variables, the cost of the gene sequencing panel test and the single-site mutation test had a much smaller effect on the incremental cost. Within the defined ranges for all the variables, the gene sequencing panel strategy was always associated with cost savings over the single-site mutation test strategy. In addition, when the proportion of patients in clinical trials was reduced from $10 \%$ to zero (and correspondingly the proportion of patients receiving ipilimumab therapy was increased from 83.4 to $93.4 \%$ ), the gene sequencing panel strategy remained associated with cost savings over the single-site mutation test strategy: the cost savings was US\$1035 when no patients were enrolled in clinical trials (Supplementary Fig. 1). Similarly, when the proportion of patients taking imatinib was reduced from $6.6 \%$ to zero, the gene sequencing panel strategy also remained associated with cost savings over the single-site mutation test strategy: the cost savings was US\$7911 when no patients took imatinib (Supplementary Fig. 2).

Since the cost of ipilimumab had the largest effect on the incremental cost of the gene sequencing panel strategy compared with the single-site mutation test strategy (Fig. 2), we also examined the effect of patients opting for a lower-cost alternative to ipilimumab therapy. As shown in Supplementary Fig. 3, as the proportion of patients opting for a lower-cost alternative therapy increased, the cost saved by the gene sequencing panel strategy decreased. However, even if $30 \%$ of patients opted for an alternative therapy that cost only $30 \%$ as much as ipilimumab therapy, the gene sequencing panel strategy was still cost saving (US\$3859 saved per patient).

Multivariable sensitivity analysis of incremental effectiveness revealed that the three variables with the greatest effects were the relative health outcome from clinical trials versus ipilimumab therapy, the proportion of patients who
Fig. 2 Multivariable sensitivity analysis for the gene sequencing panel strategy compared with the single-site mutation test strategy. Tornado plots show the effect of uncertainty of model parameters on incremental cost (a) and incremental effectiveness (b). The lower and upper bounds of the variables are listed on either side of the bars. In $\mathbf{b}$, clinical trial versus ipilimumab indicates the relative health outcome for clinical trial therapy versus ipilimumab therapy
A

Single-site mutation test positive

Cost of vemurafenib

Percentage in clinical trial

Cost of gene panel test

Cost of imatinib

Gene panel test positive

Rate of ipilimumab reinducation

Dose of ipilimumab reinduction

Cost of single-site mutation test

Percentage taking imatinib

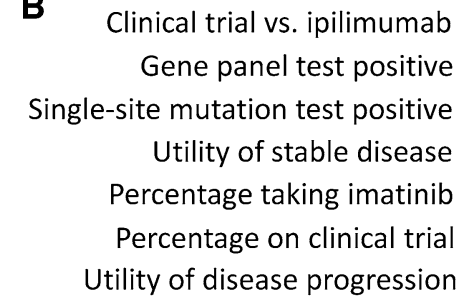

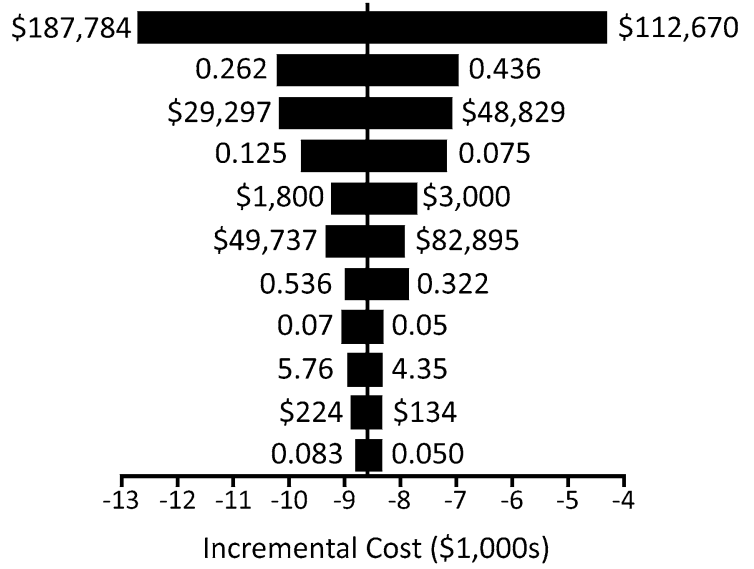

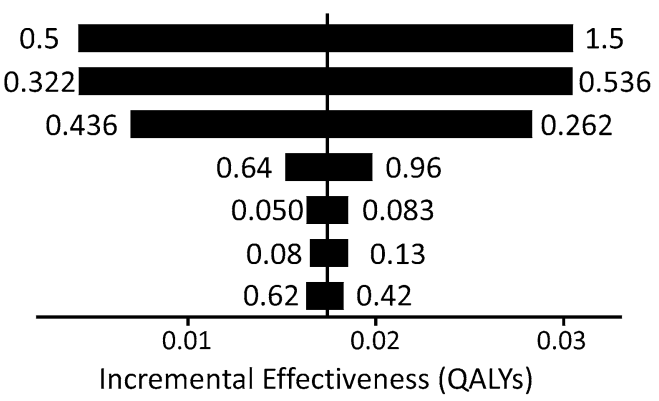




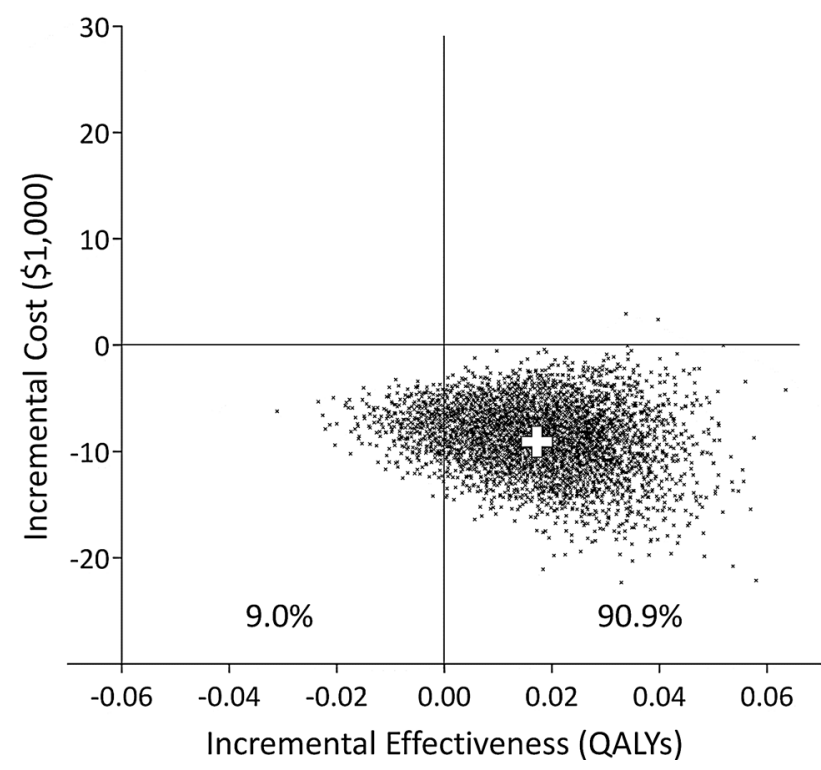

Fig. 3 Probabilistic sensitivity analysis for the gene sequencing panel strategy compared with the single-site mutation test strategy. The cost-effectiveness plane shows the effect of simultaneously varying all model parameters on incremental cost (vertical axis) and incremental effectiveness (horizontal axis) in 5000 Monte Carlo simulations. The base-case outcome is shown by a white cross

test BRAF V600 positive by the gene sequencing panel test, and the proportion of patients who test positive by the single-site mutation test (Fig. 2b). Within the defined range of these three variables, the gene sequencing panel strategy could increase the effectiveness of therapies by as much as 0.03 QALYs over the single-site mutation test strategy. The other four variables shown in Fig. $2 b$ (i.e. utility of stable disease, percentage of patients taking imatinib, percentage of patients enrolled in clinical trials, and utility of disease progression) had a smaller effect on incremental cost. Within the defined range, these variables also resulted in higher QALYs from the gene sequencing panel strategy than the single-site mutation test strategy.

In a probabilistic sensitivity analysis based on 5000 Monte Carlo simulations, the gene sequencing panel strategy was almost always associated with lower cost than the single-site mutation test strategy (Fig. 3). This analysis also revealed that the gene sequencing panel strategy had $90.9 \%$ chance of having increased QALYs compared with the single-site mutation test strategy.

\section{Discussion}

The analysis in this study showed that testing patient tumors with a 34-gene sequencing panel can lower the medical costs and increase the patient's quality of life compared with a single-site mutation test. In the base case, the gene sequencing panel strategy cost US\$8943 less and increased the QALYs by 0.0174 years per patient over a 2-year time horizon compared with the single-site mutation test strategy. If these results were applied to the $8900 \mathrm{pa}-$ tients diagnosed with metastatic melanoma in the USA each year, the gene sequencing panel strategy could result in an annual savings of US\$79.6 million and a gain of 155 QALYs. A sensitivity analysis demonstrated that the costs of the genetic tests (both the gene sequencing panel test and the single-site mutation test) had a minimal effect on the incremental cost of the gene sequencing panel strategy over the single-site mutation test strategy. Rather, the costs of ipiliumab and vemurafenib were among the most important parameters affecting the incremental cost. The incremental gain in QALYs in the gene sequencing panel strategy was primarily driven by the higher number of patients who would test $B R A F$ mutation positive. Modeling the uncertainties of the parameters showed that the gene sequencing panel strategy is most likely (i.e. $90.9 \%$ probability) to dominate the single-site mutation test strategy and thus merits consideration in the clinical management of patients with metastatic melanoma. Consistent with our model, the European Medicines Agency review of vemurafenib concluded that there was sufficient evidence to support clinical benefit for mutations other than the single nucleotide variant that results in a V600E substitution [29].

The gene sequencing panel test could help identify additional patients who might benefit from first-line BRAF inhibitor therapies; these patients would otherwise receive less effective and more costly immunotherapy. In the base case, the gene sequencing panel test identified $8 \%$ more patients with BRAF V600 mutations than did the single-site mutation test (Table 1). Recent NGS analysis of melanoma tumors has identified other BRAF mutations such as L597 mutations and the PAPSSI-BRAF fusion, which are sensitive to BRAF and MEK inhibitors [17, 30]. Therefore, the assumption that $8 \%$ more actionable $B R A F$ mutations will be identified by the gene sequencing panel test than by the single-site mutation test is likely to be an under estimate. The gene sequencing panel test could also help identify patients with other actionable mutations that respond to therapies approved for use in tumors of different origins or therapies under clinical development. For example, patients with a KIT mutation may be treated with imatinib instead of immunotherapy, and a small-molecule MEK1/2 inhibitor has shown activity against melanomas with NRAS-mutations present in $20 \%$ of the patients [31]. And the greater the difference in the mutation detection rate between the two test strategies, the greater the cost effectiveness of the gene sequencing panel strategy.

One limitation of this study is that we did not consider non-drug medical costs. However, these non-drug medical costs would most likely increase the incremental cost 
savings of gene sequencing panel strategy over the singlesite mutation test strategy. This increase in the incremental cost savings would be due to fewer patients in the gene sequencing panel strategy receiving ipilimumab, which has higher non-drug costs because it is administered intravenously and has a high rate of side effects, compared with vemurafenib, which is taken orally and has a lower rate of side effects. Side effects could also incur direct medical cost for treatment in addition to non-drug medical costs and thereby affect the relative cost effectiveness of the two testand-treat strategies.

Another limitation of our analysis is the uncertainty in several model parameters. These include the proportion of the BRAF mutation-positive patients detected by the gene sequencing panel test; for this proportion we assumed that the gene sequencing panel test and Sanger sequencing were equally sensitive in the detection of $B R A F$ mutations because a comparison of sequencing platforms showed a perfect correlation between Sanger sequencing and NGS in detecting other mutations in tumor biopsies [32]. Other uncertain parameters include the relative health outcome of patients enrolled in clinical trials in comparison with patients receiving ipilimumab therapy; we assumed that basecase patients enrolled in clinical trials would have similar outcomes to patients treated with ipilimumab therapy (the likely treatment in the comparator arm of a clinical trial). However, the sensitivity analyses suggest that the gene sequencing panel strategy typically remained more cost effective throughout the range of parameter values explored. Nevertheless, an EVPI (expected value of perfect information) analysis would be valuable to identify areas where additional information may help to reduce the uncertainty with regard to the gene sequencing panel strategy in comparison with the single-site mutation test strategy.

A further limitation is that the decision model used in our analysis reflects present guideline-supported patient management; however, the rapid accumulation of various levels of actionable evidence used by clinicians results in a diversity of clinical practice that is not captured. For example, we assumed that patients with $B R A F$ or KIT mutations would receive targeted therapies, but some of the patients might receive immunotherapy or be enrolled in a clinical trial; however, the magnitude of latter scenario is difficult to define. Patients with BRAF V600E or V600K mutations may also be treated with a combination of BRAF and MEK inhibitors (e.g. dabrafenib and trametinib). The more expensive combination therapy could affect incremental cost of the gene sequencing panel strategy over the single-site mutation test (e.g. incremental cost would be US\$88 instead of -US\$8943 if the combination therapy costs twice as much as vemurafenib); note, however, genotyping of tumors would use a different FDA-approved companion diagnostic test (THxID BRAF assay from bioMérieux) than the cobas ${ }^{\circledR} 4800$ BRAF V600 mutation test used in our analysis [7]. In addition, our decision model only considers the first-line therapies and does not include treatment following progression. Future first-line treatments of metastatic melanoma could affect the cost effectiveness of gene sequencing panel strategy versus single-site mutation test strategy.

We have taken advantage of empirical data available from randomized clinical trials to estimate parameters in the partitioned survival models. This should have allowed us to obtain good approximate counts of patients at each cycle, although results for the vemurafenib clinical trial were based on the single-site mutation test and may not be applicable to the gene sequencing panel test, because the results from these two tests are not perfectly correlated. We limited our analysis to a 2-year time horizon because longer-term survival data have not been reported for vemurafenib; however, a longer time horizon would be of interest. An alternative method would have been to estimate transition probabilities from state to state in a Markov model and extrapolate the analysis to a life-time horizon. However, this alternative method is likely to introduce more uncertainty than the analysis carried out here. We believe that a 2-year time horizon is appropriate given the low median survival time for patients with metastatic melanoma. The validity of our parameter estimates is, nevertheless, constrained by the clinical trials themselves, and we note that clinical testing of imatinib in KIT mutation carriers had a limited enrollment of only 24 patients [11].

\section{Conclusion}

Using an NGS panel of 34 genes as an aid in informing therapy decisions for metastatic melanoma is expected to reduce costs and increase QALYs compared with the single-gene, single-site mutation test. The advantage of sequencing multiple genes likely to contain functional genetic alterations should further increase as evidence for additional actionable mutations accumulates.

Acknowledgments We thank Dov Shiffman, Matthew Beer, and Charley Rowland for helpful discussions and comments on the manuscript.

Disclosures Authors YL, LAB, JJS, JJD and FMW were employees of Quest Diagnostics and author RAB was a consultant to Quest Diagnostics when this study was conducted. Author LSW had no financial relationships to report.

Author contribution All authors contributed to study concept and design; YL and LAB collected data and performed analysis; YL drafted the manuscript, and all other authors edited the manuscript. 
Open Access This article is distributed under the terms of the Creative Commons Attribution-NonCommercial 4.0 International License (http://creativecommons.org/licenses/by-nc/4.0/), which permits any noncommercial use, distribution, and reproduction in any medium, provided you give appropriate credit to the original author(s) and the source, provide a link to the Creative Commons license, and indicate if changes were made.

\section{References}

1. American Cancer Society. Cancer facts and figures 2013. New York: The American Cancer Society; 2013.

2. National Comprehensive Cancer Network. NCCN clinical practice guidelines: melanoma (version 3.2014). http://www.nccn.org. 2014.

3. Balch CM, Gershenwald JE, Soong SJ, Thompson JF, Atkins MB, Byrd DR, et al. Final version of 2009 AJCC melanoma staging and classification. J Clin Oncol. 2009;27(36):6199-206.

4. Gogas HJ, Kirkwood JM, Sondak VK. Chemotherapy for metastatic melanoma: time for a change? Cancer. 2007;109(3):455-64.

5. Hodi FS, O'Day SJ, McDermott DF, Weber RW, Sosman JA, Haanen JB, et al. Improved survival with ipilimumab in patients with metastatic melanoma. N Engl J Med. 2010;363(8):711-23.

6. McArthur GA, Chapman PB, Robert C, Larkin J, Haanen JB, Dummer R, et al. Safety and efficacy of vemurafenib in $\mathrm{BRAF}^{\mathrm{V} 600 \mathrm{E}}$ and $\mathrm{BRAF}^{\mathrm{V} 600 \mathrm{~K}}$ mutation-positive melanoma (BRIM-3): extended follow-up of a phase 3, randomised, openlabel study. Lancet Oncol. 2014;15(3):323-32.

7. Robert C, Karaszewska B, Schachter J, Rutkowski P, Mackiewicz A, Stroiakovski D, et al. Improved overall survival in melanoma with combined dabrafenib and trametinib. $\mathrm{N}$ Engl $\mathrm{J}$ Med. 2015;372(1):30-9.

8. Luke JJ, Hodi FS. Ipilimumab, vemurafenib, dabrafenib, and trametinib: synergistic competitors in the clinical management of BRAF mutant malignant melanoma. Oncologist. 2013; 18(6):717-25.

9. Hodi FS, Corless CL, Giobbie-Hurder A, Fletcher JA, Zhu M, Marino-Enriquez A, et al. Imatinib for melanomas harboring mutationally activated or amplified KIT arising on mucosal, acral, and chronically sun-damaged skin. J Clin Oncol. 2013;31(26):3182-90.

10. Guo J, Si L, Kong Y, Flaherty KT, Xu X, Zhu Y, et al. Phase II, open-label, single-arm trial of imatinib mesylate in patients with metastatic melanoma harboring c-Kit mutation or amplification. J Clin Oncol. 2011;29(21):2904-9.

11. Carvajal RD, Antonescu CR, Wolchok JD, Chapman PB, Roman RA, Teitcher J, et al. KIT as a therapeutic target in metastatic melanoma. JAMA. 2011;305(22):2327-34.

12. Menzies AM, Long GV. Recent advances in melanoma systemic therapy. BRAF inhibitors, CTLA4 antibodies and beyond. Eur J Cancer. 2013;49(15):3229-41.

13. Jeck WR, Parker J, Carson CC, Shields JM, Sambade MJ, Peters EC, et al. Targeted next generation sequencing identifies clinically actionable mutations in patients with melanoma. Pigment Cell Melanoma Res. 2014;27(4):653-63.

14. REDBOOK online database. Truven health analytics. http://sites. truvenhealth.com/redbook/. Accessed 9 March 2015.

15. Roche Molecular Systems, Inc. cobas ${ }^{\circledR} 4800$ BRAF V600 mutation test package insert. http://www.accessdata.fda.gov/cdrh_ docs/pdf11/P110020c.pdf. Accessed 20 May 2014

16. Yang H, Higgins B, Kolinsky K, Packman K, Go Z, Iyer R, et al. RG7204 (PLX4032), a selective BRAF ${ }^{\mathrm{V} 600 \mathrm{E}}$ inhibitor, displays potent antitumor activity in preclinical melanoma models. Cancer Res. 2010;70(13):5518-27.
17. Dahlman KB, Xia J, Hutchinson K, Ng C, Hucks D, Jia P, et al. $\mathrm{BRAF}^{\mathrm{L} 597}$ mutations in melanoma are associated with sensitivity to MEK inhibitors. Cancer Discov. 2012;2(9):791-7.

18. Antonescu CR, Busam KJ, Francone TD, Wong GC, Guo T, Agaram NP, et al. L576P KIT mutation in anal melanomas correlates with KIT protein expression and is sensitive to specific kinase inhibition. Int J Cancer. 2007;121(2):257-64.

19. Klein O, Clements A, Menzies AM, O'Toole S, Kefford RF, Long GV. BRAF inhibitor activity in V600R metastatic melanoma. Eur J Cancer. 2013;49(5):1073-9.

20. Centers for Medicare and Medicard Services. Clinical diagnostic laboratory fee schedule. 2014. http://www.cms.gov/Medicare/ Medicare-Fee-for-Service-Payment/ClinicalLabFeeSched/ clinlabhtml. Accessed 17 Oct 2014.

21. Beusterien KM, Szabo SM, Kotapati S, Mukherjee J, Hoos A, Hersey $\mathrm{P}$, et al. Societal preference values for advanced melanoma health states in the United Kingdom and Australia. Br J Cancer. 2009;101(3):387-9.

22. Qu K, Pan Q, Zhang X, Rodriguez L, Zhang K, Li H, et al. Detection of BRAF V600 mutations in metastatic melanoma: comparison of the Cobas 4800 and Sanger sequencing assays. J Mol Diagn. 2013;15(6):790-5.

23. National Cancer Institute. Special issue: clinical trial enrollment. NCI Cancer Bull. 2014;7(10):7.

24. Siroy AE, Boland GM, Milton DR, Roszik J, Frankian S, Malke $\mathrm{J}$, et al. Beyond BRAF: clinical mutation panel testing by nextgeneration sequencing in advanced melanoma. J Invest Dermatol. 2015;135(2):508-15.

25. National Institute for Health and Care Excellence. Vemurafenib for treating locally advanced or metastatic BRAF V600 mutationpositive malignant melanoma. 2012. http://www.nice.org.uk/ guidance/TA269. Accessed 7 Mar 2014

26. Delea TE, Amdahl J, Wang A, Amonkar MM, Thabane M. Cost effectiveness of dabrafenib as a first-line treatment in patients with BRAF v600 mutation-positive unresectable or metastatic melanoma in Canada. Pharmacoeconomics. 2015;33(4):367-80.

27. Siegel JE, Torrance GW, Russell LB, Luce BR, Weinstein MC, Gold MR. Guidelines for pharmacoeconomic studies. Recommendations from the panel on cost effectiveness in health and medicine. Panel on cost effectiveness in health and medicine. Pharmacoeconomics. 1997;11(2):159-68.

28. Barzey V, Atkins MB, Garrison LP, Asukai Y, Kotapati S, Penrod JR. Ipilimumab in 2nd line treatment of patients with advanced melanoma: a cost-effectiveness analysis. J Med Econ. 2013;16(2):202-12.

29. da Rocha Dias S, Salmonson T, van Zwieten-Boot B, Jonsson B, Marchetti S, Schellens JH, et al. The European Medicines Agency review of vemurafenib (Zelboraf ${ }^{\circledR}$ ) for the treatment of adult patients with BRAF V600 mutation-positive unresectable or metastatic melanoma: summary of the scientific assessment of the Committee for Medicinal Products for Human Use. Eur J Cancer. 2013;49(7):1654-61.

30. Johnson DB, Dahlman KH, Knol J, Gilbert J, Puzanov I, MeansPowell $\mathrm{J}$, et al. Enabling a genetically informed approach to cancer medicine: a retrospective evaluation of the impact of comprehensive tumor profiling using a targeted next-generation sequencing panel. Oncologist. 2014;19(6):616-22.

31. Ascierto PA, Schadendorf D, Berking C, Agarwala SS, van Herpen CM, Queirolo P, et al. MEK162 for patients with advanced melanoma harbouring NRAS or Val600 BRAF mutations: a non-randomised, open-label phase 2 study. Lancet Oncol. 2013;14(3):249-56.

32. Yan B, Kuick CH, Lim M, Venkataraman K, Tennakoon C, Loh E, et al. Platform comparison for evaluation of ALK protein immunohistochemical expression, genomic copy number and hotspot mutation status in neuroblastomas. PLoS One. 2014;9(9):e106575. 